\title{
Idiopathic Hypertrophic Pachymeningitis in the Craniocervical Junction
}

\author{
Jin Hyuk Bang', Keun-Tae Cho', Eo Jin Kim² \\ Departments of ${ }^{l}$ Neurosurgery and ${ }^{2}$ Pathology, Dongguk University Ilsan Hospital, Goyang, \\ Korea and Dongguk University, Seoul, Korea
}

Idiopathic hypertrophic pachymeningitis (IHP) is a rare disease, and it is characterized by chronic progressive inflammatory fibrosis and thickening of the dura mater with resultant compression of the spinal cord or neural structure without any identifiable cause. It can occur in the intracranial or spinal dura mater alone or as a craniospinal form. The spinal form is rarer than the cranial form and the craniospinal form is extremely rare. We report a rare case of IHP in the craniocervical junction involving both the cranial and spinal dura mater and discuss the diagnosis and management of the disease.

Key Words: Idiopathic hypertrophic pachymeningitis $\cdot$ Craniocervical junction $\cdot$ Diagnosis $\cdot$ Treatment

\section{INTRODUCTION}

Hypertrophic pachymeningitis (HP) is a rare disease, and it is characterized by chronic progressive inflammatory fibrosis and thickening of the dura mater with resultant compression of the spinal cord or brain ${ }^{11,18)}$. Although accessibility and imaging techniques including magnetic resonance (MR) imaging have improved recently, it can be missed or it can go unrecognized because of its indolent and non-specific symptoms $^{5}$. Syphilis, tuberculous infection, fungal infection, and autoimmune disorders such as rheumatoid arthritis or Wegener's granulomatosis can be the possible causes, ${ }^{5,71,18)}$. However, the majority of cases have no identifiable causes and are called idiopathic HP (IHP) ${ }^{11)}$. It can occur in the intracranial or spinal dura mater alone or as a craniospinal form ${ }^{18)}$. The spinal form is rarer than the cranial form and the craniospinal form is extremely rare ${ }^{7,18)}$. Only a few cases of the craniospinal form of IHP have been reported ${ }^{1,15,18)}$.

Here, we report a rare case of IHP in the craniocervical junction involving both the cranial and spinal dura mater and discuss the diagnosis and management of IHP.

- Received: July 11, 2015 • Revised: August 21, 2015

- Accepted: September 2, 2015

Corresponding Author: Keun-Tae Cho, MD, PhD

Department of Neurosurgery, Dongguk University Ilsan Hospital, 27,

Dongguk-ro, Ilsandong-gu, Goyang-si, Gyeonggi-do 10326, Korea

Tel: +82-31-961-7322, Fax: +82-31-961-7327

E-mail: ktcho21@naver.com

®This is an Open Access article distributed under the terms of the Creative

Commons Attribution Non-Commercial License (http://creativecommons.org/ licenses/by-nc/3.0/) which permits unrestricted non-commercial use, distribution and reproduction in any medium, provided the original work is properly cited.

\section{CASE REPORT}

A 67-year-old woman presented with a 3-month history of neck pain and numbness in the upper limb. She had a 1-month history of progressive gait disturbance. On neurological examination, her motor power in the lower extremity was grade $4 / 4$ and she had gait ataxia. She could walk with assistance of a cane or with the assistance other people. Deep tendon reflex was hyperreflexic but no ankle clonus was present.

MR imaging revealed spinal cord compression by a posterior epidural mass from the occiput to $\mathrm{C} 2$. The lesion showed low signal intensity on T2-weighted images, iso- to low signal intensity on T1-weighted images, and diffuse contrast enhancement with stronger peripheral enhancement on T1-weighted gadolinium-enhanced images (Fig. 1). An epidural abscess or tumor such as extradural en plaque meningioma was suspected, but a definitive diagnosis was not made. She had no fever and a normal white blood cell count, erythrocyte sedimentation rate (ESR), and C-reactive protein (CRP) were found in the blood test.

Because of worsening neurologic deficit and uncertain diagnosis, surgery was performed without further diagnostic studies. The patient underwent posterior decompression via $\mathrm{C} 1$ total laminectomy and suboccipital craniectomy. Intraoperatively, the dura was found to be diffusely thickened and compressing the underlying spinal cord. No evidence of either an abscess or tumor was found. Total excision of the lesion was attempted, but only partial excision was achieved. The dura was partially resected to the extent of bony decompression, and a duroplasty was performed.

Microscopic findings revealed densely dispersed mixed in- 

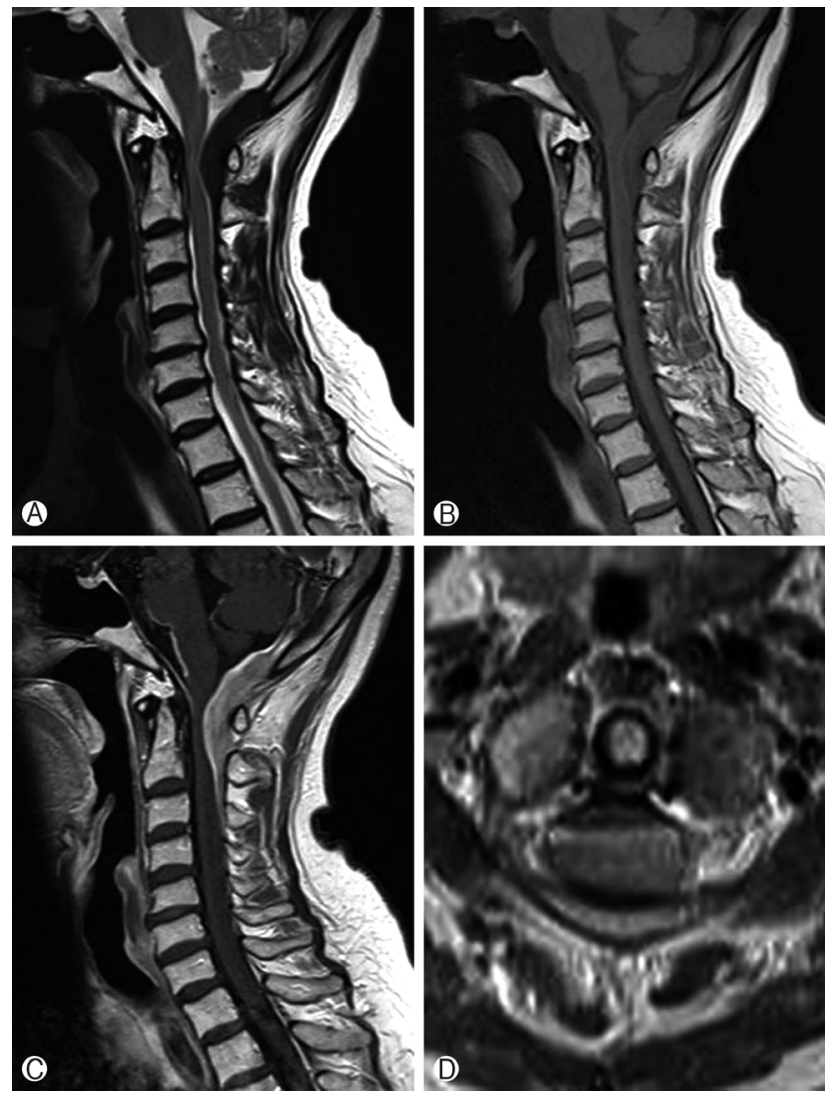

Fig. 1. Magnetic resonance images of the cervical spine. (A) T2weighted sagittal image shows an extradural mass with low signal intensity from the occiput to C2. The mass compresses the spinal cord posteriorly and high signal intensity within the spinal cord is observed. (B) The mass shows iso- to low signal intensity on T1-weighted sagittal image. Gadolinium-enhanced sagittal (C) and axial (D) images show homogeneous enhancement in the mass and stronger enhancement in the peripheral part of the mass near the subarachnoid space.

flammatory infiltrates, frequent lymphoplasmacytic cells, exuberant fibroplasia, focally scattered necrosis, and granuloma formation. There was neither an underlying pathogen responsible for infectious disease nor any evidence of other inflammatory disease (Fig. 2). Following histopathologic diagnosis, oral steroid was started. However, facial flushing and gastrointestinal discomfort developed and steroid was discontinued on the $3^{\text {rd }}$ day. Blood tests to evaluate the secondary cause of $\mathrm{HP}$ including rheumatoid factor, antinuclear antibody, and serologic test for syphilis were normal, and we concluded that this case was "idiopathic".

Postoperatively, she showed gradual improvement in her gait and was discharged 3 weeks later. She had slight numbness in the upper limb and could walk independently at the 2-yearfollow-up. However, complete recovery of walking ability was not achieved. Follow-up imaging study was not performed

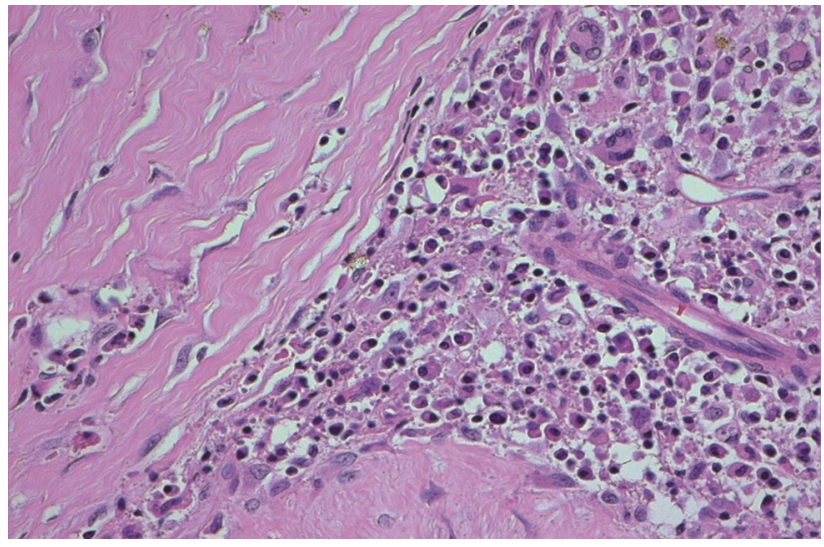

Fig. 2. Histopathologic study. Microscopic findings reveal densely dispersed mixed inflammatory infiltrates, frequent lymphoplasmacytic cells, exuberant fibroplasia, focally scattered necrosis, and granuloma formation. There is neither an underlying pathogen responsible for infectious disease nor any evidence of other inflammatory disease (Hematoxylin \& Eosin stain, x400).

because of her refusal of the study due to poor economic status and no clinical evidence of recurrence.

\section{DISCUSSION}

Craniospinal form of IHP or secondary HP is extremely rare and chief complaints and signs are chronic headache, cranial nerve palsies, cervical radiculopathy or myelopathy occurring either alone or in combination, and signs of increased intracranial pressure $(\mathrm{ICP})^{1,15-18)}$. Some authors noted the clinical courses of the patients with craniocervical HP and suggested that cranial HP extends downward to the spine ${ }^{18)}$. However, there was a case report of craniocervical IHP with no clinical and neuroradiologic evidence for this pattern ${ }^{15}$. In the present case, the patient presented with neck pain and cervical myelopathy. However, she had no symptoms or signs suggestive of cranial nerve palsy or increased ICP. Therefore, no clinical evidence of cranial $\mathrm{HP}$ extending downward to the spine was found in the present case.

In 2007, Pai et al. ${ }^{10}$ ) reviewed the MR imaging findings of the 14 published cases up to that time. In 12 of 14 patients, a long extramedullary mass of low signal intensity on T2weighted image and peripheral enhancement with central nonenhancing portion were found ${ }^{10)}$. They noted that this was a specific MR imaging finding highly suggestive of IHP in the spine $^{10)}$. This pattern represents more intense enhancement in a peripheral zone of active inflammation than in a central zone of fibrosis ${ }^{10)}$. However, homogeneous enhancement pattern in IHP is not infrequent ${ }^{3,7,12,13)}$. Hsu et al. ${ }^{3)}$ stated that homogeneous enhancement on MR image in a patient with 
IHP could be mistaken for epidural lymphoma. Low signal intensity on T2-weighted image and homogeneous enhancement pattern of the lesion were also found in spinal extradural en plaque meningioma or in diffusely calcified meningioma without dural tail sign ${ }^{8,9}$. A case of IHP with non-enhancement on T1-weighted MRI was also reported ${ }^{5)}$.

Although MR imaging is the preferred imaging modality for IHP, the diagnosis cannot be made solely based on the radiological findings ${ }^{11}$. IHP is a diagnosis of exclusion ${ }^{11}$. The diagnosis of IHP can be made when all the known etiologies including tumors such as meningioma and lymphoma, infections such as tuberculosis, sarcoidosis, or syphilis, and autoimmune disorders are ruled out and typical pathologic findings are confirmed ${ }^{11,18)}$. Biopsy is still the gold standard for the diagnosis of $\mathrm{IHP}^{11)}$. Accordingly, once the imaging study is suspicious of $\mathrm{HP}$, physicians should perform a thorough evaluation to identify or to rule out the secondary causes of $\mathrm{HP}^{11)}$.

Surgical resection is indicated when $\mathrm{HP}$ causes neurologic deficit $^{2}$. There are few case reports of the spinal form of IHP in which the treatment was started on the basis of imaging study and laboratory findings ${ }^{18)}$. In most of the reported cases, surgery was performed for pathologic diagnosis, decompression due to worsening neurologic deficit or both ${ }^{2,5,7,12,13)}$. Surgery serves a dual purpose in immediate decompression of the spinal cord and obtaining a diagnosis in patients with $\mathrm{IHP}^{12}$. In some cases, surgery was performed under the suspicion of abscess, tumor, or epidural hematoma ${ }^{7,13)}$. In most cases, complete or gross total resection was impossible. Biopsy or partial resection of the dura mater to the extent of bony decompression was possible because of the diffuse nature or long extent of $\mathrm{IHP}^{7,12,13)}$.

In the present case, our preoperative diagnosis was a tumorous condition such as en plaque meningioma. Uncertain preoperative diagnosis and progressive gait ataxia in the patient led us to perform surgery for pathologic diagnosis and decompression of the spinal cord. However, postoperative diagnosis was $\mathrm{HP}$ and the lesion was only partially resected to the extent of laminectomy and suboccipital craniectomy.

The treatment of IHP remains controversial. Steroid therapy is the mainstay of medical treatment. However, there is no clear consensus on the dose and duration of steroid therapy ${ }^{11,12)}$. In addition, radiotherapy, immunosuppressive therapy such as methotrexate and cyclophosphamide, or empirical antituberculous therapy have also been tried, but, the effect of these therapies still remains obscure ${ }^{11,15)}$. As in the above mentioned cases, surgical treatment for pathologic diagnosis and decompression of the spinal cord and subsequent steroid therapy is the optimal therapy at present ${ }^{12)}$.

Relapse or recurrence is the main problem related to long term prognosis ${ }^{24,7,18)}$. The time interval to relapse is also varia- ble from 1 week to several years after initial treatment ${ }^{4)}$. A case of relapse 17 years after the initial treatment was repor$\operatorname{ted}^{14)}$. Some authors suggested that patients with active inflammatory signs such as fever, increased ESR, increased CRP, or leukocytosis before surgery had a poor prognosis and high recurrence rate than patients without inflammatory signs ${ }^{11}$. Other authors suggested that residual pachymeningitis, arachnoiditis, or short-term use of postoperative steroid could cause rapid relapse and they recommended continuous steroid medication to avoid recurrence ${ }^{4,11,12)}$. However, there was a case report of IHP with no recurrence for 7 years after intravenous dexamethasone for only 1 week $^{6}$. In the present case, fortunately, no relapse was found clinically for 2 years in spite of residual pachymeningitis and no long-term postoperative steroid therapy. However, close observation of recurrence is necessary for a longer period.

\section{CONCLUSION}

IHP is a rare condition that can cause neurologic deficits. Suspicion of IHP, knowledge of characteristic MR imaging findings, and thorough laboratory evaluation for underlying causes are essential for accurate diagnosis. Prompt surgery for pathologic diagnosis, decompression of neural structure, or both should be considered especially when radiologic or laboratory diagnosis is uncertain or neurologic deficit presents. Postoperative steroid therapy and close monitoring of recurrence are necessary for achieving a good long-term outcome.

\section{REFERENCES}

1. Botella C, Orozco M, Navarro J, Riesgo P: Idiopathic chronic hypertrophic craniocervical pachymeningitis: case report. Neurosurgery 35:1144-1149, 1994

2. Choi HJ, Yoon DH, Lee DY, Ahn PG, Yang MS, Yi S, et al: Rapid recurrence of spinal idiopathic hypertrophic pachymeningitis. Korean J Spine 5:211-214, 2008

3. Hsu HT, Hsu SS, Chien CC, Lai PH: Teaching NeuroImages: Idiopathic hypertrophic spinal pachymeningitis mimicking epidural lymphoma. Neurology 84:e67-68, 2015

4. Ito Z, Osawa Y, Matsuyama Y, Aoki T, Harada A, Ishiguro $\mathrm{N}$ : Recurrence of hypertrophic spinal pachymeningitis. Report of two cases and review of the literature. J Neurosurg Spine 4: 509-513, 2006

5. Jee TK, Lee SH, Kim ES, Eoh W: Idiopathic hypertrophic spinal pachymeningitis with an osteolytic lesion. J Korean Neurosurg Soc 56:162-165, 2014

6. Karakasis C, Deretzi G, Rudolf J, Tsiptsios I: Long-term lack of progression after initial treatment of idiopathic hypertrophic pachymeningitis. J Clin Neurosci 19:321-323, 2012

7. Kim JH, Park YM, Chin DK: Idiopathic hypertrophic spinal pachymeningitis: report of two cases and review of the literature. 
J Korean Neurosurg Soc 50:392-395, 2011

8. Lee JW, Lee IS, Choi KU, Lee YH, Yi JH, Song JW, et al: CT and MRI findings of calcified spinal meningiomas: correlation with pathological findings. Skeletal Radiol 39:345- 352, 2010

9. Messori A, Rychlicki F, Salvolini U: Spinal epidural en-plaque meningioma with an unusual pattern of calcification in a 14year-old girl: case report and review of the literature. Neuroradiology 44:256-260, 2002

10. Pai S, Welsh CT, Patel S, Rumboldt Z: Idiopathic hypertrophic spinal pachymeningitis: report of two cases with typical MR imaging findings. Am J Neuroradiol 28:590-592, 2007

11. Qin LX, Wang CY, Hu ZP, Zeng LW, Tan LM, Zhang HN: Idiopathic hypertrophic spinal pachymeningitis: a case report and review of literature. Eur Spine J 24:S636-643, 2015

12. Ranasinghe MG, Zalatimo O, Rizk E, Specht CS, Reiter GT, Harbaugh RE, et al: Idiopathic hypertrophic spinal pachymeningitis. J Neurosurg Spine 15:195-201, 2011

13. Takahashi H, Wada A, Yokoyama Y, Ishii M, Shibuya K, Suguro $\mathrm{T}$ : Idiopathic hypertrophic spinal pachymeningitis: a case report.
J Orthop Surg (Hong Kong) 18:113-117, 2010

14. Tsutsui M, Yasuda T, Kanamori M, Hori T, Kimura T: Longterm outcome of idiopathic hypertrophic thoracic pachymeningitis. Eur Spine J 21:S404-407, 2012

15. Voller B, Vass K, Wanschitz J, Machold K, Asenbaum S, Hoberstorfer $\mathrm{M}$, et al: Hypertrophic chronic pachymeningitis as a localized immune process in the craniocervical region. Neurology 56:107-109, 2001

16. Yamashita K, Suzuki Y, Yoshizumi H, Takahashi JB, Nogawa T: Tuberculous hypertrophic pachymeningitis involving the posterior fossa and high cervical region--case report. Neurol Med Chir (Tokyo) 34:100-103, 1994

17. Yuh WT, Drew JM, Rizzo M, Ryals TJ, Sato Y, Bell WE: Evaluation of pachymeningitis by contrast-enhanced MR imaging in a patient with rheumatoid disease. Am J Neuroradiol 11:12471248, 1990

18. Zhu R, He Z, Ren Y: Idiopathic hypertrophic craniocervical pachymeningitis. Eur Spine J 24:S633-635, 2015 